\title{
Residual stress induced stabilization of martensite phase and its effect on the magnetostructural transition in Mn-rich Ni-Mn-In/Ga magnetic shape-memory alloys
}

\author{
Sanjay Singh, ${ }^{1,2}$ Pallavi Kushwaha, ${ }^{2}$ F. Scheibel, ${ }^{1}$ Hanns-Peter Liermann, ${ }^{3}$ S. R. Barman, ${ }^{4}$ M. Acet,${ }^{1}$ \\ C. Felser, ${ }^{2}$ and Dhananjai Pandey ${ }^{5}$ \\ ${ }^{1}$ Experimentalphysik, University Duisburg-Essen, D-47048 Duisburg, Germany \\ ${ }^{2}$ Max-Planck Institute for Chemical Physics of Solids, Nöthnitzer Strasse 40, 01187 Dresden, Germany \\ ${ }^{3}$ Photon Sciences, FS-PE, Deutsches Elektronen Synchrotron (DESY), 22607 Hamburg, Germany \\ ${ }^{4}$ UGC-DAE Consortium for Scientific Research, Khandwa Road, Indore, 452001 Madhya Pradesh, India \\ ${ }^{5}$ School of Materials Science and Technology, Indian Institute of Technology (Banaras Hindu University), Varanasi-221005, India
}

(Received 1 June 2015; published 22 July 2015)

\begin{abstract}
The irreversibility of the martensite transition in magnetic shape memory alloys (MSMAs) with respect to the external magnetic field is one of the biggest challenges that limits their application as giant caloric materials. This transition is a magnetostructural transition that is accompanied with a steep drop in magnetization (i.e., $\Delta M$ ) around the martensite start temperature $\left(\mathrm{M}_{\mathrm{s}}\right)$ due to the lower magnetization of the martensite phase. In this Rapid Communication, we show that $\Delta M$ around $\mathrm{M}_{\mathrm{s}}$ in $\mathrm{Mn}$-rich Ni-Mn-based MSMAs gets suppressed by two orders of magnitude in crushed powders due to the stabilization of the martensite phase at temperatures well above $\mathbf{M}_{s}$ and the austenite finish $\left(\mathrm{A}_{\mathrm{f}}\right)$ temperatures due to residual stresses. Analysis of the intensities and the FWHM of the $\mathrm{x}$-ray powder-diffraction patterns reveals stabilized martensite phase fractions as $97 \%, 75 \%$, and $90 \%$ with corresponding residual microstrains as $5.4 \%, 5.6 \%$, and $3 \%$ in crushed powders of the three different Mn-rich Ni-Mn alloys, namely, $\mathrm{Mn}_{1.8} \mathrm{Ni}_{1.8} \mathrm{In}_{0.4}, \mathrm{Mn}_{1.75} \mathrm{Ni}_{1.25} \mathrm{Ga}$, and $\mathrm{Mn}_{1.9} \mathrm{Ni}_{1.1} \mathrm{Ga}$, respectively. Even after annealing at $773 \mathrm{~K}$, the residual stress stabilized martensite phase does not fully revert to the equilibrium cubic austenite phase as the magnetostructural transition is only partially restored with a reduced value of $\Delta M$. Our results have a very significant bearing on the application of such alloys as inverse magnetocaloric and barocaloric materials.
\end{abstract}

DOI: 10.1103/PhysRevB.92.020105

PACS number(s): 81.30.Kf, 61.05.cp, 75.30.Sg

Recent years have witnessed a tremendous surge in the study of ferroic and multiferroic materials exhibiting giant caloric effects that can be used in solid-state refrigeration at temperatures close to the ambient conditions as an environmentally friendly substitute to conventional vapor compression refrigeration [1-5]. The Heusler-type Mn-rich Ni-Mn- $X(X=\mathrm{Ga}$, In, Sn, or Sb) magnetic shape memory alloys (MSMAs) have emerged as a potential family of alloys that can exhibit giant barocaloric, elastocaloric, and inverse magnetocaloric (i.e., cooling during magnetization and heating during demagnetization in contrast to normal magnetocaloric materials which heat up on magnetization and cool down by its removal) effects [6-10]. The giant inverse magnetocaloric effects (MCEs) and barocaloric effects (BCEs) in these alloys are linked with a first-order martensitic transition, which is a magnetostructural transition involving change in crystal structure as well as huge drop in magnetization $(\Delta M)$ between the austenite and the martensite phases [11]. This transition involves a large isothermal entropy change $\left(\Delta S_{\text {iso }}\right)$ and hence a large adiabatic temperature change $\left(\Delta T_{\mathrm{ad}}\right)$ that leads to the caloric effect $[8,12]$. One of the major limitations of these otherwise potentially promising Heusler alloys is the irreversibility of the martensitic transition as a function of magnetic-field cycles due to the sluggish kinetics of the first-order structural phase transition [8,13-15]. As the MSMAs owe their large inverse MCEs and BCEs due to strong coupling of magnetic and elastic degrees of freedom, the irreversibility of the $\Delta S_{\text {iso }}$ or the $\Delta T_{\text {ad }}$ with respect to magnetic field and pressure is expected to be closely related with the residual stresses and strains developed in these materials as a result of magnetic-field and/or stress (pressure) cycles.
In the present Rapid Communication, we have investigated the effect of residual stresses on the phase stabilities and magnetization behavior in two different class of $\mathrm{Mn}$-rich Ni-Mn- $X(X=\mathrm{Ga}$ and In) MSMAs whose martensite start temperature $\mathrm{M}_{\mathrm{s}}$ is close to room temperature whereas the ferromagnetic Curie temperature $\left(T_{\mathrm{c}}\right)$ is greater than $\mathrm{M}_{\mathrm{s}}$. The austenite phase of $\mathrm{Mn}$ excess Ni-Mn-In alloys is known to be ferromagnetic whereas the martensite phase is generally believed to be nonmagnetic (either paramagnetic or antiferromagnetic) $[6,8,11]$, whereas in the $\mathrm{Mn}$ excess $\mathrm{Ni}-\mathrm{Mn}-\mathrm{Ga}$ alloys, both the austenite and the martensite phases are reported to be ferrimagnetic [10,16-18]. Using high-resolution synchrotron and laboratory x-ray powder-diffraction (XRD) data, it is shown that the martensite structure can be stabilized by residual stresses over a wide temperature range well above $A_{f}$ in both alloy systems. As a direct consequence of the residual stress induced stabilization of the martensite phase, the peak value of magnetization $(M)$ around $\mathrm{M}_{\mathrm{s}}$ and the associated change in $M$ (i.e., $\Delta M$ ) between the austenite and the martensite phases is shown to decrease by two orders of magnitude. Even after annealing at $773 \mathrm{~K}$, the original value of $\Delta M$ corresponding to the bulk sample is not fully recovered due to the presence of retained martensite. Our results on Ni-Mn-In/Ga alloys unambiguously demonstrate that the residual stresses strongly affect $\Delta M$. We believe that our findings will have a very significant bearing on the application of Mn-rich Ni-Mn-type MSMAs as giant inverse magnetocaloric and barocaloric materials.

The three alloy compositions investigated by us are as follows: $\mathrm{Mn}_{1.8} \mathrm{Ni}_{1.8} \mathrm{In}_{0.4}, \mathrm{Mn}_{1.75} \mathrm{Ni}_{1.25} \mathrm{Ga}$, and $\mathrm{Mn}_{1.9} \mathrm{Ni}_{1.1} \mathrm{Ga}$. The details of sample preparation, measurements (magnetization 


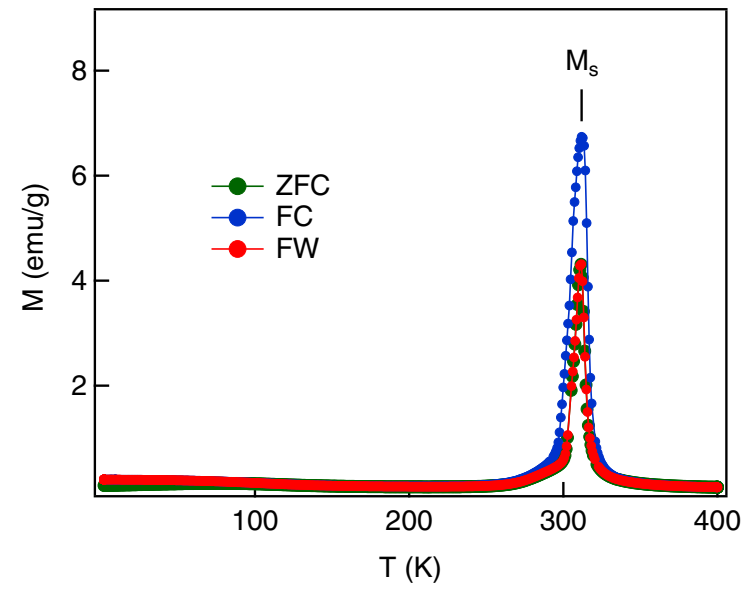

FIG. 1. (Color online) The variation in ZFC, FC, and FW magnetizations of $\mathrm{Mn}_{1.8} \mathrm{Ni}_{1.8} \mathrm{In}_{0.4}$ with temperature measured at $100 \mathrm{Oe}$. $\mathrm{M}_{\mathrm{s}}$ is the martensite start temperature.

and XRD), and Rietveld refinements are given in the Supplemental Material [19]. For the $\mathrm{Mn}_{1.8} \mathrm{Ni}_{1.8} \mathrm{In}_{0.4}$ alloy, we have investigated the magnetization and structural characteristics for annealed pieces cut from the bulk ingot, powder samples obtained by crushing the ingot, and powder samples annealed under different conditions. The results for the bulk ingot piece and crushed powder are also presented for the Ga alloys to demonstrate the likely universality of the phenomenon of the stabilization of the martensite phase due to residual stresses in Mn-rich Ni-Mn MSMAs.

The magnetization as a function of temperature $[M(T)]$ for the annealed $\mathrm{Mn}_{1.8} \mathrm{Ni}_{1.8} \mathrm{In}_{0.4}$ bulk sample (melt-ingot-annealed piece), as measured under zero-field cooling (ZFC), field cooling (FC), and field warming (FW) conditions using a magnetic field of $100 \mathrm{Oe}$, reveals a ferromagnetic transition at $T_{\mathrm{c}} \sim 316 \mathrm{~K}$ (see Fig. 1 ). The $\mathrm{M}_{\mathrm{s}}$, martensite finish $\left(\mathrm{M}_{\mathrm{f}}\right)$, and austenite start $\left(\mathrm{A}_{\mathrm{s}}\right)$ temperatures related to the austeniteto-martensitic and martensite-to-austenite transitions during cooling and warming are found to be 309,290 , and $296 \mathrm{~K}$, respectively. The low-field magnetization behavior as a function of temperature for the as-ground powder of $\mathrm{Mn}_{1.8} \mathrm{Ni}_{1.8} \mathrm{In}_{0.4}$ is drastically different from that of the annealed sample. The $M(T)$ plots recorded on the as-ground powder during cooling under 100 Oe from 300 to $2 \mathrm{~K}(\mathrm{C} 1)$, subsequent warming (at $100 \mathrm{Oe}$ ) up to $400 \mathrm{~K}(\mathrm{C} 2)$, and then cooling (at $100 \mathrm{Oe}$ ) down to $2 \mathrm{~K}$ (C3) are shown in Fig. 2. It is evident from a comparison of this figure (Fig. 2) with Fig. 1 that during the first cooling (C1) and warming (C2) cycles, the peak value of the magnetization associated with the austenite-martensite transition is about more than one order of magnitude lower than that of the annealed sample. In contrast to the $\mathrm{C} 1$ and $\mathrm{C} 2$ cycles, a comparatively larger change in magnetization associated with the austenite-martensite transition is observed when the measurement was carried out during cooling (Fig. 2, C3) the powder from $400 \mathrm{~K}$. The anomalies in the magnetization plot at the $T_{\mathrm{c}}$ and $\mathrm{M}_{\mathrm{s}}$ transitions are similar to the annealed sample (Fig. 1), but the peak value of the magnetization is still an order of magnitude lower than that for the annealed sample. Similarly an increase in the magnetization is also observed when powder was warmed from 2 to $400 \mathrm{~K}$ (C4) after the C3

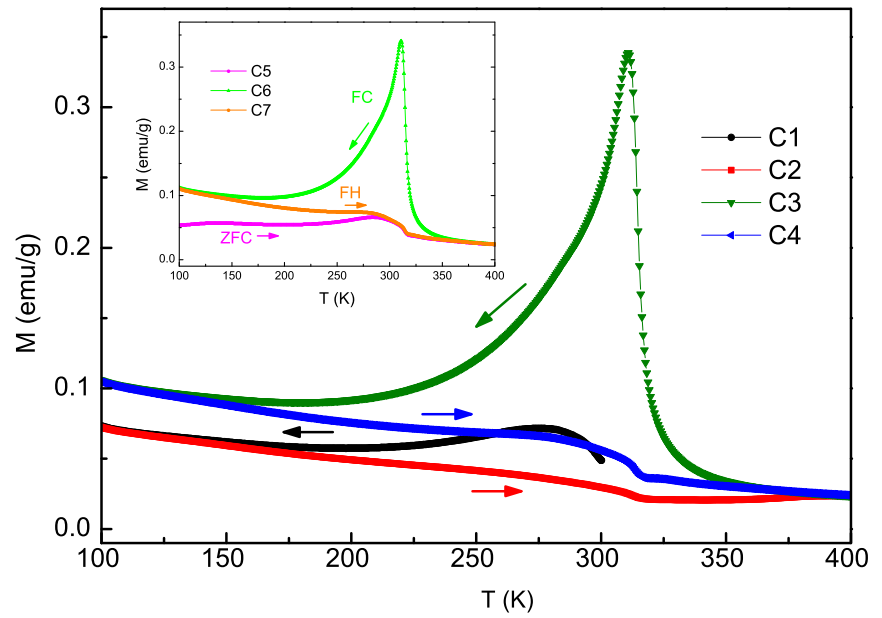

FIG. 2. (Color online) Magnetization as a function of temperature for an as-ground powder sample of $\mathrm{Mn}_{1.8} \mathrm{Ni}_{1.8} \mathrm{In}_{0.4}$ measured at $H=100 \mathrm{Oe}$ (see the text for descriptions of $\mathrm{C} 1-\mathrm{C} 4)$. The inset shows the successive data cycles taken after $\mathrm{C} 4(\mathrm{C} 5-\mathrm{C} 7)$.

cycle, but the peak value remains an order of magnitude lower than the corresponding value for the annealed bulk sample. However, with further warming and cooling cycles after $\mathrm{C} 4$ on the same powder [the inset of Fig. 2 C5 (ZFC), C6 (FC), and $\mathrm{C} 7(\mathrm{FW})]$, there was no change in the peak magnetization value at the austenite-to-martensite and reverse transitions as the $M(T)$ curve nearly coincides with those corresponding to the $\mathrm{C} 3$ and $\mathrm{C} 4$ cycles. Our results thus demonstrate that the external stresses introduced during crushing of the ingot into powder affect the magnetostructural transition drastically.

In order to understand the anomalously low values of peak magnetization of the as-ground powders as compared to that of the annealed bulk sample, we now proceed to present the details of structural studies on the as-ground and annealed samples. The powder XRD profiles of the 220 austenite peak for the as-ground and annealed $\mathrm{Mn}_{1.8} \mathrm{Ni}_{1.8} \mathrm{In}_{0.4}$ powders shown in Figs. 1(a) and 1(b) of the Supplemental Material reveals a change in crystal structure after annealing [19]. The XRD pattern for the as-ground powder of $146 \mathrm{Mn}_{1.8} \mathrm{Ni}_{1.8} \mathrm{In}_{0.4}$ was recorded at $T=340 \mathrm{~K}$, which is well above the $\mathrm{A}_{\mathrm{f}}(\sim 317 \mathrm{~K})$, estimated from the slope of the FW M-T plot in a manner similar to that adopted for determining $\mathrm{M}_{\mathrm{s}}$ from the FC M-T plot. At this temperature, the sample is expected to be in the stable austenite cubic phase (see Fig. 1). The Rietveld analysis of the XRD patterns recorded at $340 \mathrm{~K}\left(>\mathrm{M}_{\mathrm{s}}\right)$ shows that it contains $\sim 97 \%$ tetragonal martensite and $\sim 3 \%$ cubic austenite phases in the $I 4 / \mathrm{mmm}$ and $F m-3 m$ space groups, respectively [see Fig. 3(a) for the fit between observed and calculated profiles]. The tetragonal martensite phase is not expected to exist at $340 \mathrm{~K}$ as this temperature is $\sim 30 \mathrm{~K}$ higher than the $M_{s} \sim 309 K$, and it is higher than $A_{f}$. Rietveld refinement of the as-ground powder sample after annealing at $773 \mathrm{~K}$ confirms that the tetragonal phase has transformed to the thermodynamically stable cubic austenite phase [Fig. 3(b)]. Evidently, the annealed sample reveals the thermodynamically stable crystal structure whereas the as-ground sample corresponds to a stress induced martensite phase. There is, however, a very small fraction $(\sim 3 \%$ as 


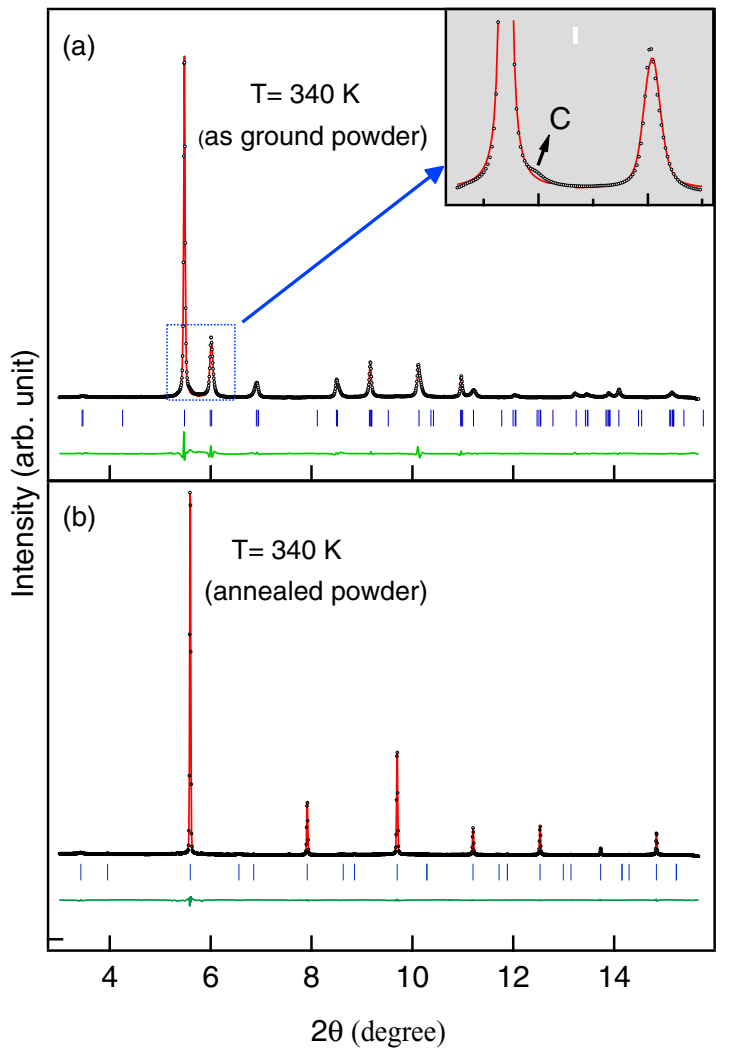

FIG. 3. (Color online) The observed (open circles), calculated (red solid line), and difference (green solid line) profiles obtained after Rietveld refinement of the structure of $\mathrm{Mn}_{1.8} \mathrm{Ni}_{1.8} \mathrm{In}_{0.4}$ considering tetragonal (space group: $14 / \mathrm{mmm}$ ) martensite and cubic austenite (space group: $F m-3 m$ ) phases: (a) as-ground powder sample and (b) after vacuum annealing the same powder at $773 \mathrm{~K}$ for $10 \mathrm{~h}$. The inset in (a) shows the presence of austenite peak (marked as C). Blue ticks represent the Bragg peak positions.

estimated from the peak intensities of the most intense peaks of the two phases) of the untransformed cubic austenite phase whose presence can also be seen in the inset of Fig. 3(a). In the case of conventional shape memory alloys, the stress induced martensite phase formed above $A_{f}$ temperature reverts back to the austenite phase on removal of the stress, and this reversibility of the austenite-martensite transition is responsible for the pseudoelastic behavior observed in those alloys $[20,21]$. In our case, the crushed sample continues to exhibit the stress induced martensite phase suggesting that the transformation is nearly irreversible in crushed powders and is therefore detrimental to caloric effects that generate stress directly through pressure [7], compressive uniaxial stress [9], or indirectly through magnetic field [11].

It is interesting to note that the Bragg peaks of the as-ground powders are very broad and become sharper after annealing (see Fig. 1 of the Supplemental Material that shows synchrotron XRD data [19]). The most likely sources of broadening in the as-ground samples are the residual stresses and the sizes of the different martensite variants. To separate out the two contributions to peak broadening, we carried out Williamson-Hall (W-H) analysis of the peak widths $(\beta)$ of the various Bragg peaks. In the Williamson-Hall analysis, the average microstrain $(\varepsilon=\langle\Delta d / d\rangle)$ and coherently scattering domain size $(\langle D\rangle)$ are determined from the relationship $\beta \cos \theta=2\langle\Delta d / d\rangle \sin \theta+\lambda /\langle D\rangle$, where $\theta$ is the Bragg angle, $\lambda$ is the wavelength of the $\mathrm{x}$ rays, and $\beta$ is the full width at half maximum (FWHM) of the peak after subtracting the FWHM of the standard sample (which in our case was $\mathrm{LaB}_{6}$ ). The $\mathrm{W}-\mathrm{H}$ plot for the as-ground powder sample of $\mathrm{Mn}_{1.8} \mathrm{Ni}_{1.8} \mathrm{In}_{0.4}$ shows two straight-line fits to the data points corresponding to various reflections (see Fig. 2 of the Supplemental Material [19]) indicating anisotropic peak broadening with the largest slope giving a residual microstrain of $\sim 5.4 \%$. The two intercepts on the ordinate axis give coherently scattering domain sizes of $\sim 43$ and $345 \AA$, respectively, corresponding to the thin martensite variants with large lateral extension (such a thin platelike morphology of the martensite phase has been reported in Ref. [22]). After annealing the powder at $773 \mathrm{~K}$, the microstrain was reduced to $0.02 \%$, and the domain size increased to $550 \AA$. The presence of large microstrains in the as-ground powder of $\mathrm{Mn}_{1.8} \mathrm{Ni}_{1.8} \mathrm{In}_{0.4}$ at $340 \mathrm{~K}$ clearly suggests that the martensite phase is stabilized by the residual stresses introduced during grinding of the ingot sample into powder. It is also interesting to note that a part of the anisotropic peak broadening is due to the change in the coherently scattering domain size which is smaller in the martensite phase due to the formation of submicron size martensite variants in thin platelike morphologies and larger in the cubic austenite phase as there are no such variants (see Table I of the Supplemental Material for the values of microstrain, domain size, and unit-cell parameters [19]).

The low peak values of magnetization at the martensiteaustenite transition for the $\mathrm{C} 1$ and $\mathrm{C} 2$ cycles in Fig. 2 is evidently linked with the stabilization of the martensite phase well above the $M_{s}$ and $A_{f}$ due to the residual stresses discussed above. In the absence of the austenite phase (fully stabilized martensite), the peak in $M(T)$ around $\mathrm{M}_{\mathrm{s}}$ is not anticipated as the peak appears due to the difference in the magnetization of the austenite and martensite phases. A small peak that appears in $M(T)$ during the $\mathrm{C} 1$ and $\mathrm{C} 2$ cycles is due to a very small fraction $(\sim 3 \%$ as determined by the relative intensity of most intense peak of the cubic austenite phase, i.e., 220) of the untransformed austenite phase. The increase in the magnetization value after taking the as-ground powder up to $400 \mathrm{~K}$ (C3 and $\mathrm{C} 4)$ is because of the increase in the fraction of the austenite phase. However, the temperature of $400 \mathrm{~K}$ is not sufficient to transform the entire martensite phase, and as a result the peak values of the magnetization at the austenite-martensite transition are still an order of magnitude lower than those for the annealed bulk samples. Even after annealing at much higher temperatures $(773 \mathrm{~K})$, the peak value for the magnetization $(\sim 3 \mathrm{emu} / \mathrm{g}$ at the martensite-austenite transition (Fig. 4) still remains only $\sim 50 \%$ of the peak value for the bulk ingot. This is because the stabilized martensite phase is still present in very small fractions in the 773-K annealed powders as can be seen from the tiny peaks in the inset of Fig. 1(b) of the Supplemental Material [19]. Our results thus show that full recovery of the magnetostructural characteristics is not possible by annealing unless the sample is recrystallized after melting.

It is interesting to note that residual stresses enhance the $\mathrm{M}_{\mathrm{s}}$ and lower the $T_{\mathrm{c}}$ (see the inset of Fig. 4, which depicts the normalized derivative plot $d M / d T$ versus $T$ ) similar to what has been reported in the studies related to barocaloric $[7,23]$ 


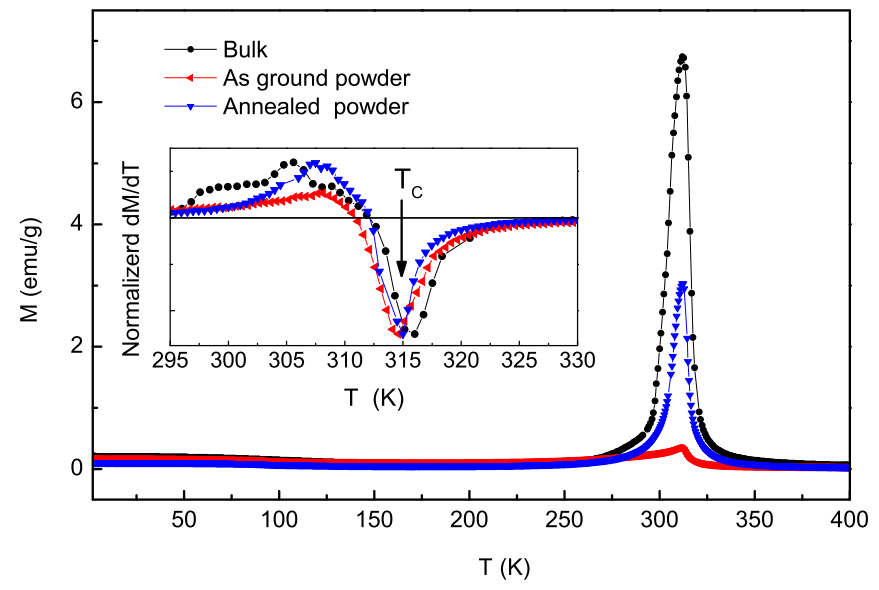

FIG. 4. (Color online) Comparison of field cooled magnetization $(H=100 \mathrm{Oe})$ as a function of temperature for annealed bulk, as-ground powder, and annealed powder (at $773 \mathrm{~K}$ ). The inset shows the normalized derivative of magnetization $(d M / d T)$. The $\mathrm{M}_{\mathrm{s}}$ values obtained from the derivative plot for annealed bulk, as-ground powder, and annealed powder are $\sim 306,308$, and $309 \mathrm{~K}$, whereas the $T_{c}^{\prime}$ s are $\sim 314.6,315$, and $316 \mathrm{~K}$, respectively.

and elastocaloric [9] effects. The increase in the magnetostructural transition temperature $\mathrm{M}_{\mathrm{s}}$ due to the residual stresses is consistent with the Clausius-Clapeyron equation " $d T / d p=$ $\Delta V / \Delta S$ " (here $\Delta S$ and $\Delta V$ are the entropy and volume changes, respectively, at the phase transition) for a first-order phase transition [7,23]. In the present case, the residual stresses play the role of pressure which not only enhance the $M_{s}$, but also stabilizes the martensite phase well above $\mathrm{M}_{\mathrm{s}}$ and $\mathrm{A}_{\mathrm{f}}$ in the powder samples due to its lower volume as compared to that of the austenite phase. The opposite behavior of $T_{\mathrm{c}}$ indicates reduced ferromagnetic exchange interaction in the plastically deformed cubic austenite regions that did not transform due to deformation. Our results therefore have a direct bearing on the giant barocaloric and elastocaloric behaviors of such alloys as the pressure may not only stabilize the martensite phase well above the $\mathrm{M}_{\mathrm{s}}$ and $\mathrm{A}_{\mathrm{f}}$ temperatures and reduce the peak value of the bulk magnetization at the magnetostructural transition temperature, but also make the transition partially irreversible. A similar irreversibility of the magnetostructural phase transition induced by the magnetic field is also anticipated because of the internal strains generated by the magnetic field [11], and this may adversely affect the magnetocaloric properties as well.

The residual stress induced stabilization of the martensite phase is not limited to In-based Ni-Mn MSMAs as we have observed similar effects in two other Mn excess Ni-Mn-Ga alloys, $\mathrm{Mn}_{1.75} \mathrm{Ni}_{1.25} \mathrm{Ga}$ and $\mathrm{Mn}_{1.9} \mathrm{Ni}_{1.1} \mathrm{Ga}$, which also show inverse magnetocaloric effect [10]. The $M(T)$ plot in a low applied magnetic field of 50 Oe for annealed bulk samples shown in Fig. 5(a) reveals $\mathrm{M}_{\mathrm{s}}, \mathrm{M}_{\mathrm{f}}, \mathrm{A}_{\mathrm{s}}$, and $\mathrm{A}_{\mathrm{f}}$ for $\mathrm{Mn}_{1.75} \mathrm{Ni}_{1.25} \mathrm{Ga}$ as $139,134,160$, and $175 \mathrm{~K}$, respectively, whereas the corresponding temperatures for $\mathrm{Mn}_{1.9} \mathrm{Ni}_{1.1} \mathrm{Ga}$ are $264,160,230$, and $315 \mathrm{~K}$. The drop at $\mathrm{M}_{\mathrm{s}}$ is related to the large magnetocrystalline anisotropy and lower magnetization of the martensite phase in these MSMAs [10,18,24]. A comparison of $\mathrm{x}$-ray powder-diffraction profiles of the as-ground powder samples and the same powder after it was annealed at
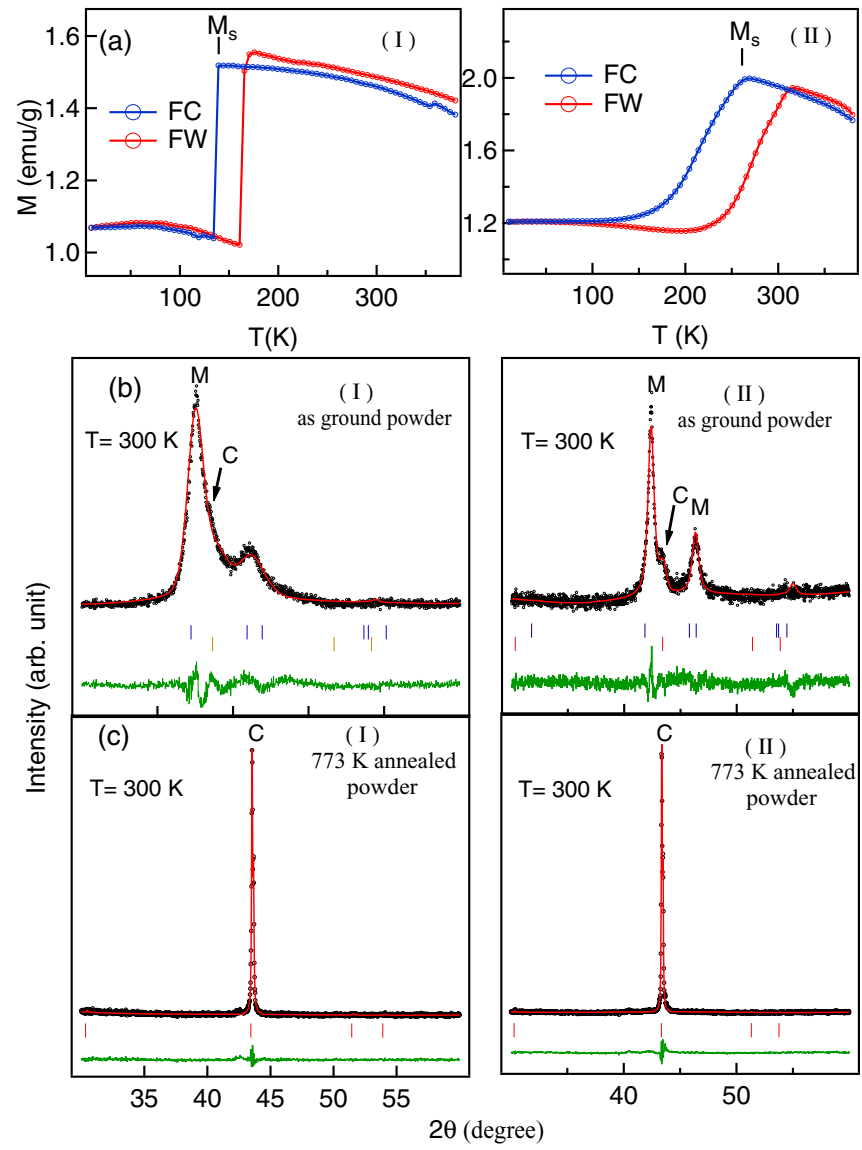

FIG. 5. (Color online) (a) Magnetization as a function of temperature, (b) Rietveld fits for a few selected peaks for as-ground powder samples, and (c) after annealing the same powder at $773 \mathrm{~K}$ for $10 \mathrm{~h}$. The labels I and II correspond to the results for $\mathrm{Mn}_{1.75} \mathrm{Ni}_{1.25} \mathrm{Ga}$ and $\mathrm{Mn}_{1.9} \mathrm{Ni}_{1.1} \mathrm{Ga}$ shown in (a)-(c). The magnetization measurements involved a magnetic field of 50 Oe during cooling (FC) and warming $(\mathrm{FW})$. The martensite start temperature $\mathrm{M}_{\mathrm{s}}$ is marked in (a). C and $M$ represent peaks related to cubic austenite and martensite phases, respectively, in (b) and (c).

$773 \mathrm{~K}$ for $10 \mathrm{~h}$ (Fig. 5) as was performed for $\mathrm{Mn}_{1.8} \mathrm{Ni}_{1.8} \mathrm{In}_{0.4}$ (Fig. 3) shows that the martensite phase has been stabilized in these alloys also at room temperature, even though the room temperature is considerably higher than $\mathrm{M}_{\mathrm{s}}$ as compared to the Ni-Mn-In alloy system due to stresses introduced during crushing. Figure 5(b) shows the Rietveld refinement results for the as-ground powder samples whereas Fig. 5(c) shows the Rietveld refinement results for the annealed powder samples. The Rietveld refinements reveal that the as-ground $\mathrm{Mn}_{1.75} \mathrm{Ni}_{1.25} \mathrm{Ga}$ and $\mathrm{Mn}_{1.9} \mathrm{Ni}_{1.1} \mathrm{Ga}$ have tetragonal structures (space group $I 4 / \mathrm{mmm}$ ) with some retained cubic (space group Fm-3m) austenite phases that correspond to $\sim 25 \%$ and $10 \%$ for $\mathrm{Mn}_{1.75} \mathrm{Ni}_{1.25} \mathrm{Ga}$ and $\mathrm{Mn}_{1.9} \mathrm{Ni}_{1.1} \mathrm{Ga}$, respectively (see Table I of the Supplemental Material for more details [19]). The Rietveld refinements of the powder samples after annealing at $773 \mathrm{~K}$ confirm that the tetragonal phase [Fig. 5(b)] reverts to the thermodynamically stable cubic austenite phase after annealing [Fig. 5(c)]. The W-H analysis shows large residual microstrains of about $5.6 \%$ and $3 \%$ in the as-ground powder samples of $\mathrm{Mn}_{1.75} \mathrm{Ni}_{1.25} \mathrm{Ga}$ and $\mathrm{Mn}_{1.9} \mathrm{Ni}_{1.1} \mathrm{Ga}$, which are 
reduced to $0.5 \%$ and $0.35 \%$, respectively, after annealing the powder at $773 \mathrm{~K}$ in the cubic austenite phase [Fig. 5(c)]. A similar effect has also been observed for $\mathrm{Mn}_{2} \mathrm{NiGa}$ [25] where the martensitic phase is stabilized at temperatures higher than $\mathrm{M}_{\mathrm{s}}$ and $\mathrm{A}_{\mathrm{f}}$.

In conclusion, we have shown that the Mn-rich Ni-Mnbased MSMAs are highly sensitive to residual stresses, which can stabilize the martensite phase far above the martensite transition temperature. The fact that this effect is observed for both In- and Ga-based Ni-Mn- $X$ alloys shows that this may not be related with the active element ( $X=\mathrm{In}$ or $\mathrm{Ga}$ ) and that it is the excess $\mathrm{Mn}$ that is crucial to the stabilization of the martensite phase well above the martensite start and austenite finish temperatures in these systems. This stabilization drastically affects the characteristics of the magnetostructural transition because of the lower magnetization of the martensite phase and will therefore have an important bearing on applications of these alloys as magnetocaloric and barocaloric materials.

S.S. thanks the Alexander von Humboldt Foundation, Germany for a Fellowship. D.P. acknowledges the financial support for the $\mathrm{X}$-ray diffraction studies using synchrotron radiation under the DST-DESY Project operated through the Saha Institute of Nuclear Physics and Science and Engineering Research Board of India for financial support through the award of a J. C. Bose National Fellowship.
[1] O. Tegus, E. Brück, K. H. J. Buschow, and F. R. de Boer, Transition-metal-based magnetic refrigerants for roomtemperature applications, Nature (London) 415, 150 (2002).

[2] L. Manosa, A. Planes, and M. Acet, Advanced materials for solid-state refrigeration, J. Mater. Chem. A 1, 4925 (2013).

[3] X. Moya, S. Kar-Narayan, and N. D. Mathur, Caloric materials near ferroic phase transitions, Nature Mater. 13, 439 (2014).

[4] F. Guillou, G. Porcari, H. Yibole, N. V. Dijk, and E. Brück, Taming the first-order transition in giant magnetocaloric materials, Adv. Mater. 26, 2671 (2014).

[5] S. Fähler, U. K. Rößler, O. Kastner, J. Eckert, G. Eggeler, H. Emmerich, P. Entel, S. Müller, E. Quandt, and K. Albe, Caloric effects in ferroic materials: New concepts for cooling, Adv. Eng. Mater. 14, 10 (2012).

[6] T. Krenke, M. Acet, E. F. Wassermann, X. Moya, L. Manosa, and A. Planes, Inverse magnetocaloric effect in ferromagnetic Ni-Mn-Sn alloys, Nature Mater. 4, 450 (2005).

[7] L. Manosa, D. Gonzalez-Alonso, E. Bonnot, M. Barrio, J. Tamarit, S. Aksoy, and M. Acet, Giant solid-state barocaloric effect in the Ni-Mn-In magnetic shape-memory alloy, Nature Mater. 9, 478 (2010).

[8] J. Liu, T. Gottschal, K. P. Skokov, James, D. Moore, and O. Gutfleisch, Giant magnetocaloric effect driven by structural transitions, Nature Mater. 11, 620 (2012).

[9] Y. J. Huang, Q. D. Hu, N. M. Bruno, J.-H. Chen, I. Karaman, J. H. Ross, and J. G. Li, Giant elastocaloric effect in directionally solidified Ni-Mn-In magnetic shape memory alloy, Scr. Mater. 105, 42 (2015).

[10] S. Singh, S. E. Muthu, A. Senyshyn, P. Rajput, E. Suard, S. Arumugam, and S. R. Barman, Inverse magnetocaloric effect in $\mathrm{Mn}_{2} \mathrm{NiGa}$ and $\mathrm{Mn}_{1.75} \mathrm{Ni}_{1.25} \mathrm{Ga}$ magnetic shape memory alloys, Appl. Phys. Lett. 104, 051905 (2014).

[11] R. Kainuma, Y. Imano, W. Ito, Y. Sutou, H. Morito, S. Okamoto, O. Kitakami, K. Oikawa, A. Fujita, T. Kanomata, and K. Ishida, Magnetic-field-induced shape recovery by reverse phase transformation, Nature (London) 439, 957 (2006).

[12] T. Kihara, X. Xu, W. Ito, R. Kainuma, and M. Tokunaga, Direct measurements of inverse magnetocaloric effects in metamagnetic shape-memory alloy NiCoMnIn, Phys. Rev. B 90, 214409 (2014).

[13] I. Titov, M. Acet, M. Farle, D. Gonzalez-Alonso, L. Manosa, A. Planes, and T. Krenke, Hysteresis effects in the inverse magnetocaloric effect in martensitic Ni-Mn-In and Ni-Mn-Sn, J. Appl. Phys. 112, 073914 (2012).

[14] M. G. Zavareh, C. S. Mejía, A. K. Nayak, Y. Skourski, J. Wosnitza, C. Felser, and M. Nicklas, Direct measurements of the magnetocaloric effect in pulsed magnetic fields: The example of the Heusler alloy $\mathrm{Ni}_{50} \mathrm{Mn}_{35} \mathrm{In}_{15}$, Appl. Phys. Lett. 106, 071904 (2015).

[15] A. K. Nayak, C. S. Mejia, S. W. D’Souza, S. Chadov, Y. Skourski, C. Felser, and M. Nicklas, Large field-induced irreversibility in Ni-Mn based Heusler shape-memory alloys: A pulsed magnetic field study, Phys. Rev. B 90, 220408(R) (2014).

[16] G. D. Liu, X. F. Dai, S. Y. Yu, Z. Y. Zhu, J. L. Chen, G. H. Wu, H. Zhu, and J. Q. Xiao, Physical and electronic structure and magnetism of $\mathrm{Mn}_{2} \mathrm{NiGa}$ : Experiment and densityfunctional theory calculations, Phys. Rev. B 74, 054435 (2006).

[17] S. R. Barman and A. Chakrabarti, Comment on "Physical and electronic structure and magnetism of $\mathrm{Mn}_{2} \mathrm{NiGa}$ : Experiment and density-functional theory calculations", Phys. Rev. B 77, 176401 (2008).

[18] S. Singh, R. Rawat, S. E. Muthu, S. W. D'Souza, E. Suard, A. Senyshyn, S. Banik, P. Rajput, S. Bhardwaj, A. M. Awasthi, R. Ranjan, S. Arumugam, D. L. Schlagel, T. A. Lograsso, A. Chakrabarti, and S. R. Barman, Spin-Valve-Like Magnetoresistance in $\mathrm{Mn}_{2} \mathrm{NiGa}$ at room temperature, Phys. Rev. Lett. 109, 246601 (2012).

[19] See Supplemental Material at http://link.aps.org/supplemental/ 10.1103/PhysRevB.92.020105 for details of sample preparation, measurements, Williamson-Hall analysis, and Rietveld refinements.

[20] K. Otsuka and C. M. Wayman, Shape Memory Materials (Cambridge University Press, Cambridge, U.K., 1998); K. Otsuka and T. Kakeshita, Science and technology of shape-memory alloys: New developments, Mater. Res. Bull. 27, 91 (2002).

[21] K. Ullako, J. K. Huang, C. Kantner, R. C. OHandley, and V. V. Kokorin, Large magnetic-field-induced strains in $\mathrm{Ni}_{2} \mathrm{MnGa}$ single crystals, Appl. Phys. Lett. 69, 1966 (1996).

[22] Y. Kishi, Z. Yajima, K. Shimizu, and M. Wuttig, Transformation behavior and microstructures of PtNiMnGa ferromagnetic shape memory alloys, Mater. Sci. Eng., A 378, 361 (2004).

[23] L. Mañosa, X. Moya, A. Planes, O. Gutfleisch, J. Lyubina, M. Barrio, J.-L. Tamarit, S. Aksoy, T. Krenke, and M. Acet, Effects of hydrostatic pressure on the magnetism and martensitic 
transition of Ni-Mn-In magnetic superelastic alloys, Appl. Phys. Lett. 92, 012515 (2008).

[24] F. Albertini, L. Pareti, and A. Paoluzi, L. Morellon, P. A. Algarabel, M. R. Ibarra, and L. Righi, Composition and temperature dependence of the magnetocrystalline anisotropy in
$\mathrm{Ni}_{2+x} \mathrm{Mn}_{1+y} \mathrm{Ga}_{1+z}(x+y+z=0)$ Heusler alloys, Appl. Phys. Lett. 81, 4032 (2002).

[25] S. Singh M. Maniraj, S. W. D’Souza, R. Ranjan, and S. R. Barman, Structural transformations in $\mathrm{Mn}_{2} \mathrm{NiGa}$ due to residual stress, Appl. Phys. Lett. 96, 081904 (2010). 